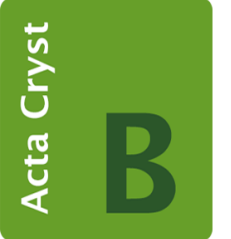

STRUCTURAL SCIENCE

CRYSTAL ENGINEERING

MATERIALS

Volume 74 (2018)

Supporting information for article:

Hydrophobic dipeptides: the final piece in the puzzle Carl Henrik Görbitz 


\section{Hydrophobic dipeptides: the final piece in the puzzle}

\section{Carl Henrik Görbitz}

Department of Chemistry, University of Oslo, Norway

Supporting information

\section{Figure S1}

Standard and non-standard $\mathbf{S 4}$ hydrogen bonding pattern

Figure S2

Selection of anti hydrogen bonding patterns

\section{Figure S3}

S5/T5 hybrid hydrogen bonding pattern in the crystal structure of Phe-Ile 0.88 hydrate

\section{Table S1}

Hydrogen bonding patterns in the crystal structures of hydrophobic dipeptides (short version)

\section{Table S2}

Hydrogen bonding patterns in the crystal structures of hydrophobic dipeptides (extended version including redeterminations and pseudopolymorphs)

\section{Table S3}

Hydrogen bonding patterns in the crystal structures of all dipeptides

\section{Table S4}

Torsion angles in dipeptide structures for the main structural classes 


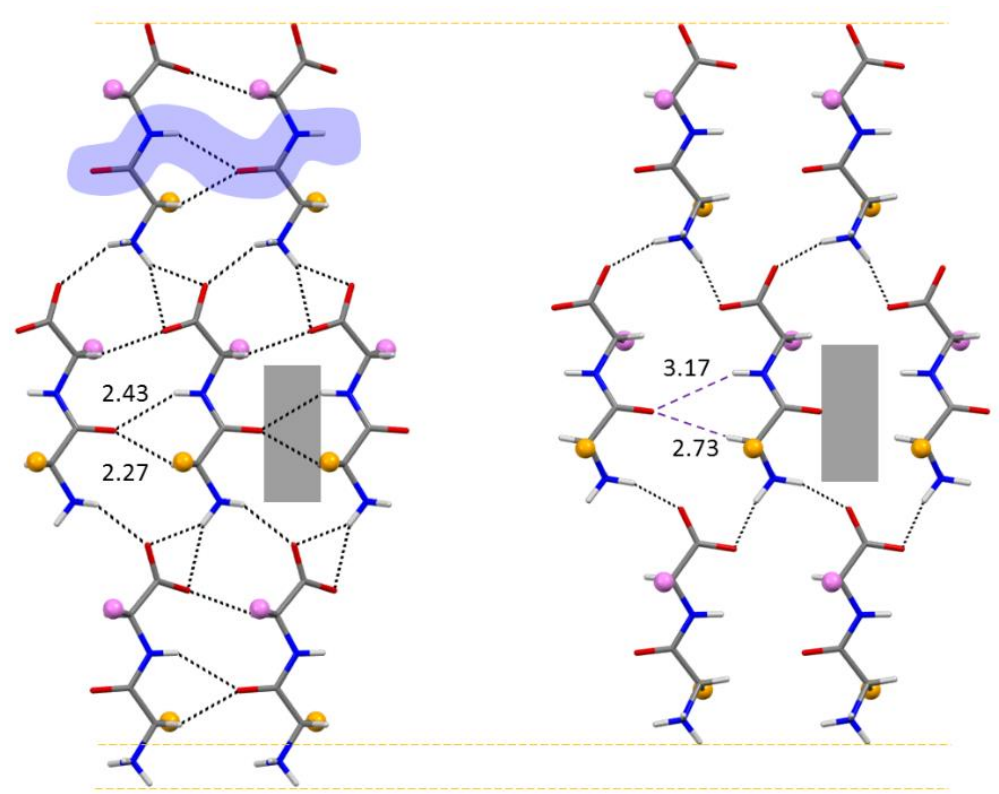

(a)

(b)

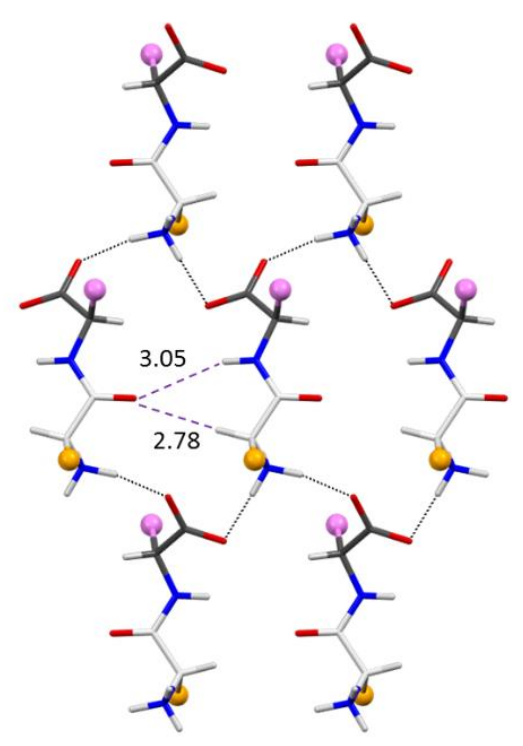

(c)

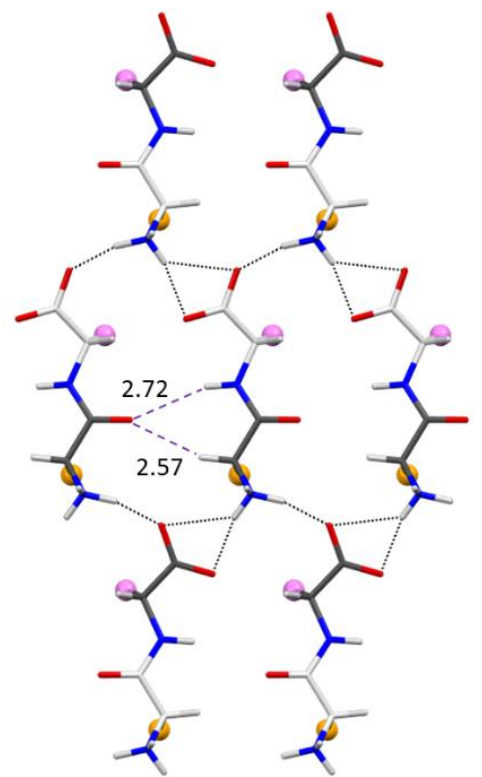

(d)

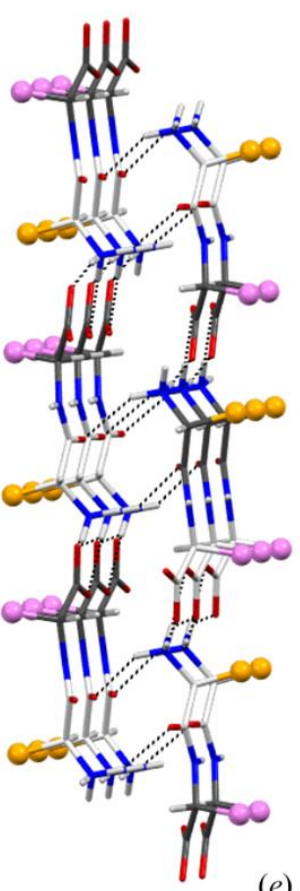

(e)

\section{Figure S1}

(a) Standard S4 hydrogen bonding pattern in the structure of Phe-Phe methanol solvate (CSD refcode JOQLIM)(Mason et al., 2014) with a $C(4)$ chain involving the amide $>\mathrm{N}-\mathrm{H}$ donor (blue shade). There is also a $C(4)$ chain involving the neighbouring $\mathrm{C}^{\alpha}-\mathrm{H}$ donor. $(b)$ Due to the presence of a large cocrystallized molecule in D-fGly-D-fGly $(R)$-2-chlorophenyl methyl sulfoxide clathrate (XEJQIM, in the illustration inverted to the L-L enantiomer)(Akazome et al., 2000), head-to tail chains are more widely separated (grey box) so that $\mathrm{H} \cdots \mathrm{O}$ distances (given in $\AA$ after normalization of $\mathrm{N}-\mathrm{H}$ covalent bonds to $0.88 \AA$ and $\mathrm{C}-\mathrm{H}$ bonds to $1.00 \AA$ ) become long for hydrogen bonds. The relationship with the structure in $(a)$ is nevertheless obvious, so this is still referred to as a $\mathbf{S 4}$ pattern. Expansion along the horizontal axis leads to contraction along the vertical axis, as indicated by the dashed, orange lines. (c) Hydrogen bonding in $(S)$-fGly- $(R)$-fGly formamide solvate (Akazome et al., 2002). C-atoms of Land D-amino acids are coloured in light and dark grey, respectively. The peptide conformation is rather folded, and the sheet is isolated in the crystal structure. (d) $\mathbf{S 4}$ hydrogen bonding in the racemate LD-Ala-DL-Met (Murali et al., 1986), chirality colour code as in (c). Two such sheets, related by a center-of-symmetry, make a hydrophilic layer in the structure, as seen edge-on in $(e)$. 

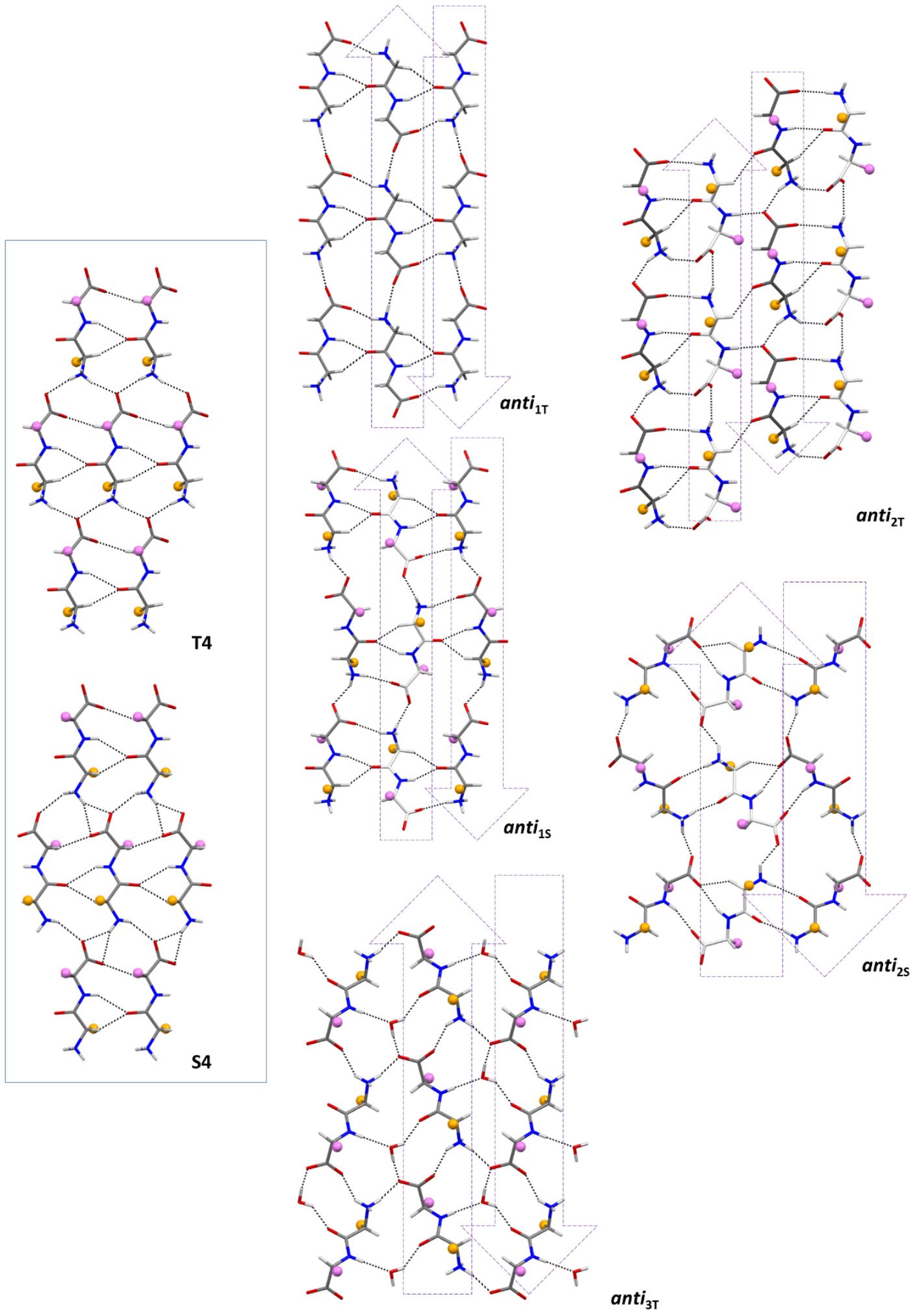

\section{Figure S2}

Selection of anti hydrogen bonding patterns. The subscript gives a running number and relationship to one of the standard patterns $\mathbf{T 4}$ or $\mathbf{S 4}$ (inside box). The $\boldsymbol{a n t i}_{1 \mathbf{T}}$ (observed only for $\alpha$-Gly-Gly)(Kvick et al., 1977, Biswas et al., 1968) and anti $\boldsymbol{i}_{1 \mathrm{~s}}$ patterns can be derived from the standard patterns by flipping every second head-to-tail chain in the opposite direction, as indicated by the large, open arrows. In

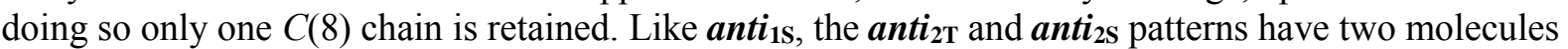
in the asymmetric unit (drawn with light and dark grey C-atoms), while anti $\mathbf{i}_{3 \mathrm{~T}}$ has an integrated water molecule. 


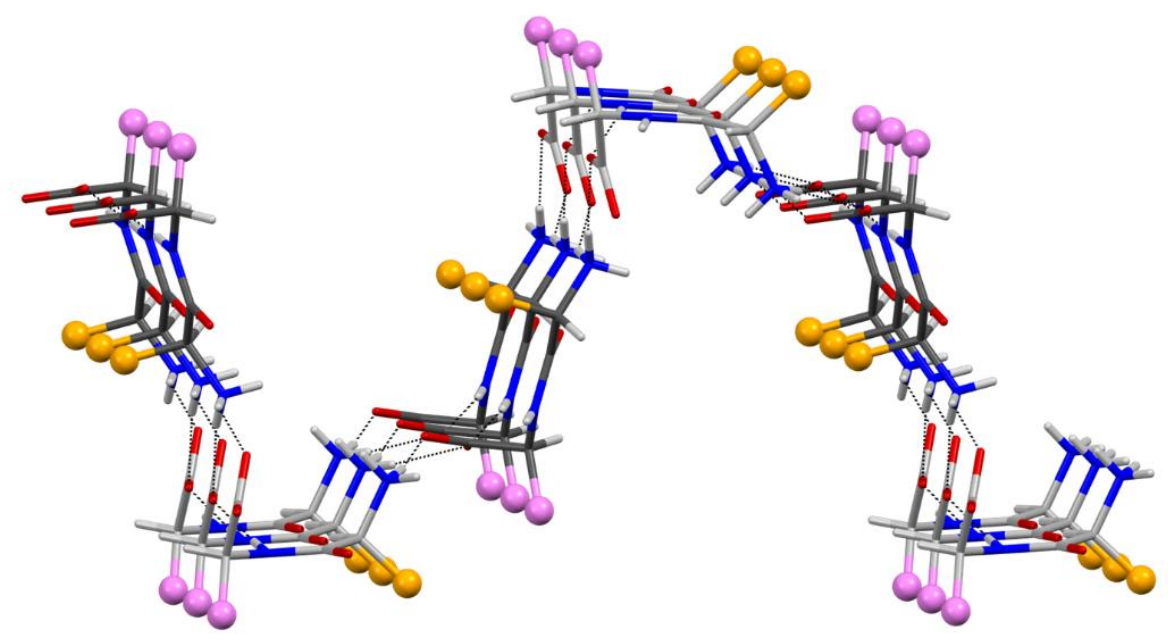

(a)

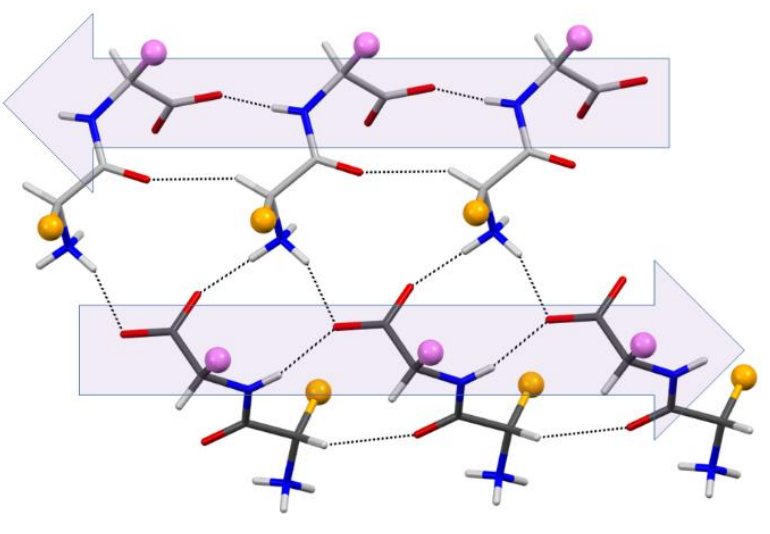

(b)

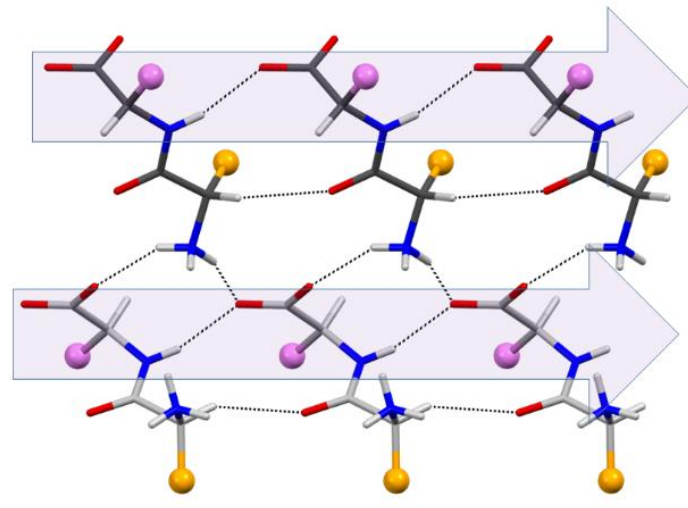

(c)

\section{Figure S3}

S5/T5 hybrid hydrogen bonding pattern in the crystal structure of Phe-Ile 0.88 hydrate (PAJPUM)(Görbitz, 2004b). (a) Hydrogen bonds between the main chains of the two molecules in the asymmetric unit (light and dark grey C-atoms, respectively) generate a strongly undulating 2-

dimensional sheet. (b) One interface between rows of molecules in $C(5)$ chains showing characteristics of a S5 pattern in that neighbouring chains (large, open arrows) are antiparallel.

(c) Second interface showing characteristics of a $\mathbf{T 5}$ pattern with parallel $C(5)$ chains. 
Table S1

Hydrogen bonding patterns in the crystal structures of hydrophobic dipeptides. ${ }^{a}$

\begin{tabular}{|c|c|c|c|c|c|c|c|}
\hline Sequence $^{b}$ & Refcode $^{c}$ & Comment & Packing ${ }^{d}$ & Sp. gr. ${ }^{*}$ & $Z^{\prime}$ & $T(\mathrm{~K})$ & Year \\
\hline \multicolumn{8}{|c|}{ Peptides with combinations of Ala, Val, Ile, Leu and Phe } \\
\hline Ala-Ala & ALAALA & & T5-t & 14 & 1 & 295 & 1971 \\
\hline \multirow[t]{2}{*}{ Ala-Val } & XUDVOH & & VA & $P 6_{1}$ & 1 & 150 & 2002 \\
\hline & XUDWAU & 0.25 2-propanol $0.22 \mathrm{H} 2 \mathrm{O}$ & VA & $P 6_{1}$ & 4 & 150 & 2002 \\
\hline Ala-lle & AQAROZ & $0.47 \mathrm{H}_{2} \mathrm{O}$ & VA & $P 6_{1}$ & 1 & 150 & 2003 \\
\hline Ala-Leu & DEZQOO & $0.50 \mathrm{H}_{2} \mathrm{O}$ & T5 & $C 2$ & 1 & 150 & 1999 \\
\hline Ala-Phe & COCGEG & 2.00 2-propanol, A mode ${ }^{t}$ & S5 & $P 2_{1} 2_{1} 2_{1}$ & 1 & 150 & 1999 \\
\hline \multirow[t]{2}{*}{ Val-Ala } & WIRYEB & & VA & $P 6_{1}$ & 1 & 120 & 1996 \\
\hline & NAYZET & 0.35 acetonitrile $0.29 \mathrm{H}_{2} \mathrm{O}$ & VA & $P 2_{1}$ & 3 & 105 & 2005 \\
\hline Val-Val & AQASIU & $1.03 \mathrm{H}_{2} \mathrm{O}$ & VA & $P 6_{1}$ & 1 & 150 & 2003 \\
\hline Val-Ile & AQASEQ & $0.22 \mathrm{H}_{2} \mathrm{O}$ & VA & $P 6_{1}$ & 1 & 105 & 2003 \\
\hline Val-Leu & (1) this work & 1.00 acetonitrile, $\mathbf{A}$ mode & S5 & $P 2_{1} 2_{1} 2_{1}$ & 1 & 105 & 2018 \\
\hline \multirow[t]{3}{*}{ Val-Phe } & COCGIK & 1.00 2-propanol & \$4 & $P 2_{1}$ & 4 & 150 & 1999 \\
\hline & MOBYAD & $2.00 \mathrm{H}_{2} \mathrm{O}$, similar to Nva-Phe (VIKWUJ) & $\mathrm{T}^{* *}$ & $P 2_{1} 2_{1} 2_{1}$ & 1 & 150 & 2002 \\
\hline & MOBYEH & $3.00 \mathrm{H}_{2} \mathrm{O}$ & 0 & $P 2_{1}$ & 8 & 150 & 2002 \\
\hline Ile-Ala & AQARUF & & VA & $P 6_{1}$ & 1 & 105 & 2003 \\
\hline Ile-Val & AQASAM & $0.21 \mathrm{H}_{2} \mathrm{O}$ & VA & $P 6_{1}$ & 1 & 105 & 2003 \\
\hline Ile-lle & YAGZOW & $2.00 \mathrm{H}_{2} \mathrm{O}$ & $(\mathbf{S 5})$ & $P 2_{1} 2_{1} 2_{1}$ & 1 & 105 & 2004 \\
\hline Ile-Leu & ETITUW & $0.91 \mathrm{H}_{2} \mathrm{O}$ & T5-t & $C 2$ & 2 & 105 & 2004 \\
\hline Ile-Phe & ETONIK & $2.00 \mathrm{H}_{2} \mathrm{O}$ & $\mathrm{T}^{* *}$ & $P 2_{1}$ & 1 & 105 & 2004 \\
\hline \multirow[t]{3}{*}{ Leu-Ala } & TELVOV & $1.00 \mathrm{DMSO}$ & \$4 & $P 2_{1} 2_{1} 2_{1}$ & 1 & 295 & 1996 \\
\hline & FEHPAK & 1.00 benzyl methyl sulfoxide & S4 & $P 2_{1}$ & 1 & 298 & 2005 \\
\hline & RAVMOQ & $4.00 \mathrm{H}_{2} \mathrm{O}$ & $\mathrm{S5}^{*}$ & $P 2{ }_{1} 2_{1} 2_{1}$ & 1 & 150 & 1997 \\
\hline \multirow[t]{4}{*}{ Leu-Val } & NAFZID & $0.75 \mathrm{H}_{2} \mathrm{O}$, similar to Leu-lle (ETIWIN) & 0 & $P 6_{2}$ & 4 & 120 & 1996 \\
\hline & JUCSEF01 & 1.00 2-propanol, Ac mode & S5 & $P 2_{1} 2_{1} 2_{1}$ & 1 & 150 & 1999 \\
\hline & SUWLIF & 1.00 methanol & S5 & $P 2_{1}$ & 1 & 150 & 1999 \\
\hline & SUWLOL & 1.00 ethanol & S5 & $P 2_{1}$ & 4 & 150 & 1999 \\
\hline \multirow[t]{3}{*}{ Leu-lle } & ETIWIN & $0.75 \mathrm{H}_{2} \mathrm{O}$, similar to Leu-Val (NAFZID) & 0 & $P 6_{2}$ & 4 & 105 & 2004 \\
\hline & $\mathrm{HIZCOJ}$ & $2.50 \mathrm{H}_{2} \mathrm{O}$ & 0 & $P 2_{1} 2_{1} 2_{1}$ & 2 & 110 & 2008 \\
\hline & IКОМОМ & 1.00 trifluoroethanol & S5 & $P 2_{1}$ & 2 & 120 & 2016 \\
\hline \multirow[t]{4}{*}{ Leu-Leu } & YORPEA & 1.00 DMSO & S5 & $P 2_{1}$ & 1 & 295 & 1994 \\
\hline & HIQWAF & 1.00 2-methyl-1-propanol, A mode, $Z^{\prime}=2$ & S5 & $P 2_{1} 2_{1} 2_{1}$ & 2 & 150 & 1999 \\
\hline & IDUZOW & $0.87 \mathrm{H}_{2} \mathrm{O}$ & T5-t & $P 2_{1} 2_{1} 2_{1}$ & 2 & 150 & 2001 \\
\hline & JUQQIV & 1.00 ethanol & anti $_{2 \pi}$ & $P 2_{1}$ & 2 & 150 & 1998 \\
\hline \multirow[t]{2}{*}{ Leu-Phe } & COCGOQ & 1.00 2-propanol $0.10 \mathrm{H}_{2} \mathrm{O}$ & S4 & $P 2_{1}$ & 2 & 150 & 1999 \\
\hline & IDUZUC & $0.86 \mathrm{H}_{2} \mathrm{O}$ & T5-t & $P 2_{1}$ & 2 & 150 & 2001 \\
\hline Phe-Ala & QIMBUJ & $2.00 \mathrm{H}_{2} \mathrm{O}$ & screw & $P 2_{1} 2_{1} 2_{1}$ & 1 & 150 & 2001 \\
\hline Phe-Val & XEGNAY & & S5 & $P 2_{1} 2_{1} 2_{1}$ & 1 & 150 & 2000 \\
\hline Phe-lle & PAJPUM & $0.88 \mathrm{H}_{2} \mathrm{O}$ & S5/T5 & $P 2_{1}$ & 2 & 105 & 2004 \\
\hline Phe-Leu & IFABAS & $1.26 \mathrm{H}_{2} \mathrm{O}$ & T5-t & $P 2_{1} 2_{1} 2_{1}$ & 2 & 150 & 2001 \\
\hline \multirow[t]{2}{*}{ Phe-Phe } & IFABEW & $2.47 \mathrm{H}_{2} \mathrm{O}$ & T5-t & $P 6_{1}$ & 1 & 150 & 2001 \\
\hline & JOQLIM & 2.00 methanol & S4 & $P 2_{1} 2_{1} 2_{1}$ & 1 & 298 & 2014 \\
\hline \multicolumn{8}{|c|}{ Other hydrophobic dipeptides with S5 sheets } \\
\hline Phe*-Phe* & ITOBEA & & S5 & $P 2_{1}$ & 3 & 100 & 2016 \\
\hline Ile-fGly & OKOZIY & 1.00 methyl o-tolyl sulfoxide, $\mathbf{A}$ mode & S5 & $P 22_{1} 2_{1}$ & 1 & 173 & 2010 \\
\hline \multirow[t]{2}{*}{ fGly-fGly } & SAMWUZ & 1.0 (4-bromobenzyl)methylsulfoxide & S5 & $P 2_{1}$ & 1 & 173 & 2004 \\
\hline & SAMWOT & 1.0 (4-fluorobenzyl)methylsulfoxide, A mode & S5 & $P 2_{1} 2_{1} 2_{1}$ & 1 & 173 & 2004 \\
\hline bCys-bCys & SBLCYS & Bc mode & S5 & $P 2_{1} 2_{1} 2_{1}$ & 1 & 295 & 1975 \\
\hline
\end{tabular}

${ }^{a}$ Excluding redeterminations and pseudopolymorphs. ${ }^{b} \mathrm{Phe}{ }^{*}=$ Phe with $-\mathrm{CH}_{2}$ - replaced by $-\mathrm{C}_{2} \mathrm{H}_{4^{-}}$, fGly = phenylglycine; bCys $=\mathrm{S}$-benzylcysteine. ${ }^{\mathrm{c}}$ Rows in grey shade with refcodes in bold share $\mathbf{S} 5$ pattern and $P 2_{1} 2_{1} 2_{1}$ space group with (I) presented here. ${ }^{d}$ Designators from Figure 1 of the main body of the paper; yellow, blue and red fill colour indicates structural family as in Fig. 6(a). VA is the nanotubular Val-Ala class, while O means absence of a clear pattern. The S5* pattern of Ile-lle dehydrate (YAGZOW)(Görbitz, 2004a) is only tentative and has been put in parenthesis. For Phe-Ala (QIMBUJ)(Görbitz, 2001) "screw" means presence of a single, isolated $C(8)$ chain of molecules related by a screw axis. e Space group. ${ }^{f}$ The various modes are described in (Görbitz, 2010). 
Table S2

Hydrogen bonding patterns in the crystal structures of hydrophobic dipeptides [1]

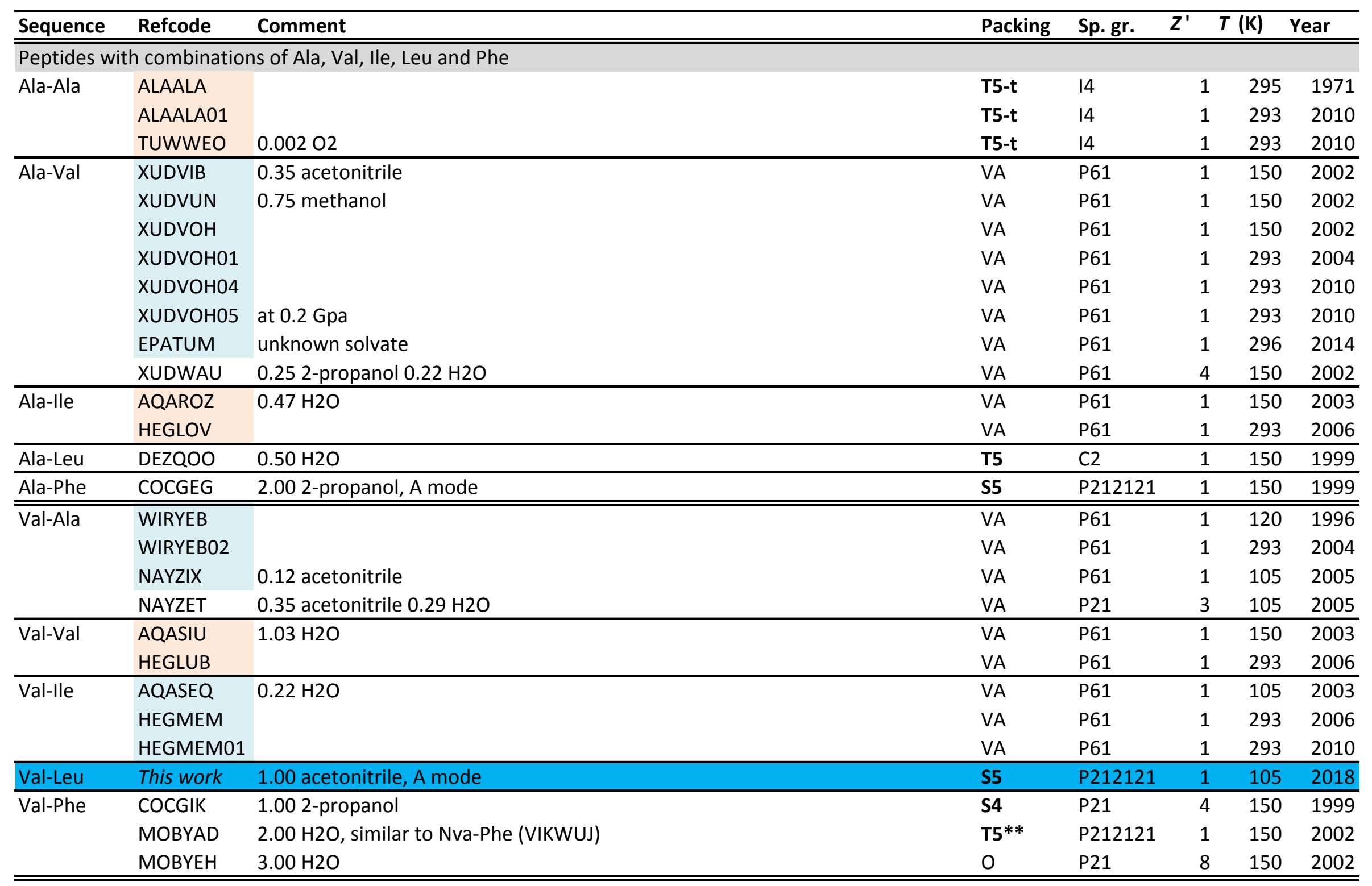




\begin{tabular}{|c|c|c|c|c|c|c|c|}
\hline \multirow[t]{2}{*}{ Ile-Ala } & AQARUF & & VA & P61 & 1 & 105 & 2003 \\
\hline & AQARUF01 & & VA & P61 & 1 & 293 & 2006 \\
\hline \multirow[t]{2}{*}{ Ile-Val } & AQASAM & $0.21 \mathrm{H} 2 \mathrm{O}$ & VA & P61 & 1 & 105 & 2003 \\
\hline & HEGMAI & & VA & P61 & 1 & 293 & 2006 \\
\hline Ile-Ile & YAGZOW & $2.00 \mathrm{H} 2 \mathrm{O}$ & $(\mathbf{S} 5 *)$ & $\mathrm{P} 212121$ & 1 & 105 & 2004 \\
\hline Ile-Leu & ETITUW & $0.91 \mathrm{H} 2 \mathrm{O}$ & T5-t & $\mathrm{C} 2$ & 2 & 105 & 2004 \\
\hline Ile-Phe & ETONIK & $2.00 \mathrm{H} 2 \mathrm{O}$ & T5** & P21 & 1 & 105 & 2004 \\
\hline \multirow[t]{5}{*}{ Leu-Ala } & TELVOV & $1.00 \mathrm{DMSO}$ & S4 & P212121 & 1 & 295 & 1996 \\
\hline & TELVOV01 & 1.00 DMSO & S4 & P212121 & 1 & 298 & 2005 \\
\hline & FEHPEO & 1.00 isobutyl methyl sulfoxide & S4 & P212121 & 1 & 298 & 2005 \\
\hline & FEHPAK & 1.00 benzyl methyl sulfoxide & S4 & P21 & 1 & 298 & 2005 \\
\hline & RAVMOQ & $4.00 \mathrm{H} 2 \mathrm{O}$ & S5* & P212121 & 1 & 150 & 1997 \\
\hline \multirow[t]{4}{*}{ Leu-Val } & NAFZID & $0.75 \mathrm{H} 2 \mathrm{O}$, similar to Leu-lle (ETIWIN) & 0 & P62 & 4 & 120 & 1996 \\
\hline & JUCSEF01 & 1.00 2-propanol, Ac mode & S5 & P212121 & 1 & 150 & 1999 \\
\hline & SUWLIF & 1.00 methanol & S5 & P21 & 1 & 150 & 1999 \\
\hline & SUWLOL & 1.00 ethanol & S5 & P21 & 4 & 150 & 1999 \\
\hline \multirow[t]{3}{*}{ Leu-Ile } & ETIWIN & $0.75 \mathrm{H} 2 \mathrm{O}$, similar to Leu-Val (NAFZID) & 0 & P62 & 4 & 105 & 2004 \\
\hline & HIZCOJ & $2.50 \mathrm{H} 2 \mathrm{O}$ & $\mathrm{O}$ & P212121 & 2 & 110 & 2008 \\
\hline & IKOMOM & 1.00 trifluoroethanol & S5 & P21 & 2 & 120 & 2016 \\
\hline \multirow[t]{7}{*}{ Leu-Leu } & YORPEA & $1.00 \mathrm{DMSO}$ & S5 & $\mathrm{P} 21$ & 1 & 295 & 1994 \\
\hline & HIQWAF & 1.00 2-methyl-1-propanol, A mode, $Z^{\prime}=2$ & S5 & P212121 & 2 & 150 & 1999 \\
\hline & IDUZOW & $0.87 \mathrm{H} 2 \mathrm{O}$ & T5-t & P212121 & 2 & 150 & 2001 \\
\hline & JUQQIV & 1.00 ethanol & anti-2T & P21 & 2 & 150 & 1998 \\
\hline & JUQQOB & 1.00 1-propanol & anti- $2 \mathrm{~T}$ & P21 & 2 & 150 & 1998 \\
\hline & JUQQUH & 1.00 2-propanol & anti- $2 \mathrm{~T}$ & P21 & 2 & 150 & 1998 \\
\hline & JUQRAO & 0.55 2-propanol 0.45 1-propanol & anti- $2 \mathrm{~T}$ & P21 & 2 & 150 & 1998 \\
\hline \multirow[t]{2}{*}{ Leu-Phe } & COCGOQ & 1.002 -propanol & S4 & P21 & 2 & 150 & 1999 \\
\hline & IDUZUC & $0.86 \mathrm{H} 2 \mathrm{O}$ & T5-t & P21 & 2 & 150 & 2001 \\
\hline Phe-Ala & QIMBUJ & $2.00 \mathrm{H} 2 \mathrm{O}$ & screw & P212121 & 1 & 150 & 2001 \\
\hline Phe-Val & XEGNAY & & S5 & P212121 & 1 & 150 & 2000 \\
\hline Phe-Ile & PAJPUM & $0.88 \mathrm{H} 2 \mathrm{O}$ & S5/T5 & P21 & 2 & 105 & 2004 \\
\hline Phe-Leu & IFABAS & $1.26 \mathrm{H} 2 \mathrm{O}$ & T5-t & P212121 & 2 & 150 & 2001 \\
\hline
\end{tabular}




\begin{tabular}{|c|c|c|c|c|c|c|c|}
\hline \multirow[t]{4}{*}{ Phe-Phe } & IFABEW & $2.47 \mathrm{H} 2 \mathrm{O}$ & T5-t & P61 & 1 & 150 & 2001 \\
\hline & OREVAK & $1.00 \mathrm{H} 2 \mathrm{O}$ (PXRD) & T5-t & P61 & 1 & 298 & 2014 \\
\hline & JOQLEI & $1.60 \mathrm{H} 2 \mathrm{O}$ & T5-t & P61 & 1 & 298 & 2014 \\
\hline & JOQLIM & 2.00 methanol & S4 & P212121 & 1 & 298 & 2014 \\
\hline \multicolumn{8}{|c|}{ Peptides with Met } \\
\hline Ala-Met & EMIPAR & $0.5 \mathrm{H} 2 \mathrm{O}$ & S4/T5 & P212121 & 2 & 105 & 2003 \\
\hline Met-Ala & CAQTOD & 1.0 2-propanol & S4 & $\mathrm{P} 21$ & 2 & 150 & 2000 \\
\hline Met-Ala & OLOGEB & & VA & P61 & 7 & 105 & 2003 \\
\hline Met-Met & METMET & & S4 & P212121 & 1 & 295 & 1975 \\
\hline \multicolumn{8}{|c|}{ Peptides with Trp } \\
\hline Ala-Trp & FUJZUF & $1.0 \mathrm{H} 2 \mathrm{O}$ & 0 & P212121 & 1 & 295 & 2000 \\
\hline Phe-Trp & GEHTAP & $0.75 \mathrm{H} 2 \mathrm{O}$ & T5-t & P212121 & 4 & 105 & 2006 \\
\hline Ile-Trp & BEQJAJ & $2.0 \mathrm{H} 2 \mathrm{O}$, similar to Val-Phe (MOBYAD) & T5** & P212121 & 1 & 193 & 2004 \\
\hline Trp-Val & BEQJEN & $2.0 \mathrm{H} 2 \mathrm{O}$ & S5** & $\mathrm{P} 212121$ & 1 & 193 & 2004 \\
\hline \multicolumn{8}{|c|}{ VA-class peptides with Abu and/or Nva } \\
\hline Nva-Val & GAGFIG & & VA & P61 & 1 & 105 & 2015 \\
\hline Nva-Leu & GAGFOM & & VA & P61 & 1 & 105 & 2015 \\
\hline Nva-Ile & GAGFUS & & VA & P61 & 1 & 105 & 2015 \\
\hline Abu-Abu & GAGPAI & & VA & P61 & 1 & 105 & 2015 \\
\hline Abu-Nva & GAGPEM & & VA & P61 & 1 & 105 & 2015 \\
\hline Abu-Val & GAGPIQ & & VA & P61 & 1 & 105 & 2015 \\
\hline Abu-Leu & GAGPOW & & VA & P61 & 1 & 105 & 2015 \\
\hline Abu-lle & GAGPUC & & VA & P61 & 1 & 105 & 2015 \\
\hline Nva-Abu & GAGQAJ & & VA & P61 & 1 & 105 & 2015 \\
\hline Nva-Nva & GAGQEN & & VA & P61 & 1 & 105 & 2015 \\
\hline \multicolumn{8}{|c|}{ Other peptides with Abu or Nva } \\
\hline Ala-Abu & JAYFOF & & $\mathrm{T} 5 / \mathrm{T} 5 / \mathrm{T} 4$ & P21 & 3 & 105 & 2005 \\
\hline Ala-Abu & XOSHUI & $0.33 \mathrm{H} 2 \mathrm{O}$ & $\mathrm{T} 5 / \mathrm{T} 5 / \mathrm{T} 4$ & $\mathrm{P} 21$ & 3 & 293 & 2002 \\
\hline Abu-Ala & XOSHOC & & T5-t & 14 & 1 & 293 & 2002 \\
\hline Nva-Phe & VIKWUJ & $2.00 \mathrm{H} 2 \mathrm{O}$, similar to Val-Phe (MOBYAD) & $\mathrm{T} 5 * *$ & P212121 & 1 & 105 & 2013 \\
\hline
\end{tabular}


Petides with Phe derivatives

\begin{tabular}{|c|c|c|c|c|c|c|c|}
\hline Phe*-Phe* & ITOBEA & & S5 & $\mathrm{P} 21$ & 3 & 100 & 2016 \\
\hline Phe*-Phe* & ITOBIE & 0.5 trifluoroacetate & anti & P212121 & 4 & 100 & 2016 \\
\hline Phe*-Phe* & ІтОВОК & 0.25 trifluoroacetate $0.25 \mathrm{H} 3 \mathrm{O}+0.125 \mathrm{H} 2 \mathrm{O}$ & anti & $\mathrm{P} 21$ & 8 & 100 & 2016 \\
\hline \multicolumn{8}{|c|}{ Peptides with fGly and/or nGly } \\
\hline Ile-fGly & OKOZAQ & 1.00 methyl phenyl sulfoxide & S4 & P212121 & 1 & 173 & 2010 \\
\hline Ile-fGly & OKOZIY & 1.00 methyl o-tolyl sulfoxide, A mode, layer slide & S5 & P212121 & 1 & 173 & 2010 \\
\hline Leu-fGly & OKOZEU & 1.00 methyl 3,4-dimethylphenyl sulfoxide & S4 & $\mathrm{P} 212121$ & 1 & 173 & 2010 \\
\hline \multirow[t]{19}{*}{ nGly-fGly } & MAVTEI & $1.0(S)$-ethyl lactate $R R$ & anti-2S & $\mathrm{P} 21$ & 2 & 295 & 1999 \\
\hline & MAVTIM & 0.5 pantolactone 1.0 methanol $0.5 \mathrm{H} 2 \mathrm{O} R R$ & anti-2S & P21 & 2 & 173 & 1999 \\
\hline & MAVVOU & 1.0 (S)-methyl 2-hydroxy-3-methylbutyrate $R R$ & anti-2S & $\mathrm{P} 21$ & 2 & 295 & 1999 \\
\hline & RUGHOQ & 1.02 .0 methanol $1.0 \mathrm{H} 20 R R$ conformationally distinct from MAVTEI & anti-2S & P21 & 2 & 173 & 1996 \\
\hline & DABQOM & 1.0 1,2-dimethoxybenzene $R R$ & S4 & P212121 & 1 & 295 & 1997 \\
\hline & RUGHIK & 1.02 .0 methanol $1.0 \mathrm{H} 20 R R$ layer slide compared to DABQOM & S4 & P212121 & 1 & 173 & 1996 \\
\hline & GUYSUO & $1.01,2$ diethyl ether $1.0 \mathrm{H} 2 \mathrm{O} R R$ & anti-3T & P212121 & 1 & 298 & 2002 \\
\hline & GUYTAV & 1.0 1,2-dimethoxyethane $1.0 \mathrm{H} 2 \mathrm{O} R R$ & anti-3T & P212121 & 1 & 298 & 2002 \\
\hline & GUYTEZ & 1.0 1-methoxy-2-methylthioethane $1.0 \mathrm{H} 20 \mathrm{O} R$ & anti-3T & P212121 & 1 & 298 & 2002 \\
\hline & GUYTID & 1.0 1-methoxyethane $1.0 \mathrm{H} 2 \mathrm{O} R R$ & anti-3T & P212121 & 1 & 298 & 2002 \\
\hline & GUYTOJ & 1.0 1,2-dimethoxypropane $1.0 \mathrm{H} 2 \mathrm{O} R R$ & anti-3T & P212121 & 1 & 298 & 2002 \\
\hline & GUYTUP & 1.0 1,3-dimethoxypropane $1.0 \mathrm{H} 2 \mathrm{O} R R$ & anti-3T & P212121 & 1 & 298 & 2002 \\
\hline & MAVTAE & $1.0(S)$-methyl lactate $R R$ & anti-2S & P212121 & 2 & 295 & 1999 \\
\hline & MAVTOS & 0.5 methyl 2-hydroxyvalerate 1.0 methanol $R R$ & anti-2S & P212121 & 2 & 173 & 1999 \\
\hline & MAVTUY & 0.5 methyl (S)-2-hydroxyhexanoate 1.0 methanol $R R$ & anti-2S & P212121 & 2 & 173 & 1999 \\
\hline & MAVVAG & 0.5 (S)-methyl 2-hydroxy-4-methylvalerate 1.0 methanol $R R$ & anti-2S & P212121 & 2 & 295 & 1999 \\
\hline & MAVVEK & $0.5(S)$-methyl mandelate $1.0 \mathrm{H} 2 \mathrm{O} R R$ & anti-2S & P212121 & 2 & 295 & 1999 \\
\hline & MAVVIO & 0.5 methyl 2-hydroxy-3-methylbutyrate 1.0 methanol $0.5 \mathrm{H} 20 R R$ & anti-1S & P212121 & 2 & 173 & 1999 \\
\hline & MAVVUA & 0.5 (S)-methyl 2-hydroxy-3,3-dimethylbutyrate 1.0 methanol $0.5 \mathrm{H} 2 \mathrm{O} R R$ & anti-1S & P212121 & 2 & 173 & 1999 \\
\hline \multirow[t]{6}{*}{ fGly-fGly } & XEJQOS & 1.0 rac-benzyl methyl sulfoxide $R R$ & S4 & P1 & 2 & 295 & 2000 \\
\hline & SAMWUZ & 1.0 (4-bromobenzyl)methylsulfoxide $R R$ & S5 & P21 & 1 & 173 & 2004 \\
\hline & SAMXAG & 1.0 (4-iodobenzyl)methylsulfoxide $R R$ & S5 & P21 & 1 & 298 & 2004 \\
\hline & SAMWOT & 1.0 (4-fluorobenzyl)methylsulfoxide $R R$, A mode & S5 & P212121 & 1 & 173 & 2004 \\
\hline & XEJQEI & 1.0 (S)-methyl 3-tolyl sulfoxide $R R$ & S4 & P212121 & 1 & 295 & 2000 \\
\hline & XEJQIM & $1.0(R)$-2-chlorophenyl methyl sulfoxide $R R$ & S4 & P212121 & 1 & 295 & 2000 \\
\hline
\end{tabular}




\begin{tabular}{|c|c|c|c|c|c|c|c|}
\hline & JENTOL01 & $1.00(S)$-isopropylphenylsulfoxide $R R$ & S4 & P212121 & 1 & 295 & 1997 \\
\hline & NOTSEU & $1.00(S)$-ethyl phenyl sulfoxide $R R$ & S4 & P212121 & 1 & 295 & 1997 \\
\hline & NOTSIY & $1.00(S)$-ethyl phenyl sulfoxide $R R$ layer slide compared to NOTSEU & S4 & P21212 & 1 & 295 & 1997 \\
\hline & WILTIV & $S R$ & T5 & P21 & 1 & 298 & 2007 \\
\hline & WILTOB & 1.00 diethylsulfoxide $S R$ & S4 & P21 & 1 & 298 & 2007 \\
\hline & HUZVON & 1.00 dimethylformamide $S R$ & S4 & P212121 & 1 & 173 & 2002 \\
\hline & HUZVUT & 1.00 acetamide clathrate $S R$ & S4 & P212121 & 1 & 298 & 2002 \\
\hline & HUZWAA & $1.00 \mathrm{~N}, \mathrm{~N}$-dimethylacetamide $S R$ & S4 & P212121 & 1 & 298 & 2002 \\
\hline & WINZEZ & 1.00 dimethylsulfoxide $S R$ & S4 & P212121 & 1 & 298 & 2007 \\
\hline & WILTUH & 1.00 ethyl-methylsulfoxide $S R$ layer slide from HUZVON/WINZEZ & S4 & P212121 & 1 & 298 & 2007 \\
\hline \multicolumn{8}{|c|}{ Other hydrophobic dipeptides } \\
\hline bCys-bCys & SBLCYS & Bc mode & S5 & P212121 & 1 & 295 & 1975 \\
\hline
\end{tabular}

[1] Legends as in Table S1 with addition of further codes for anti patterns taken from Fig. S2 (the patterns for the Phe*-Phe* structures ITOBIE and ITOBOK, with high Z'-values, are unique to these structures). Alternating light blue and gold sets of refcodes identify groups of essentially identical structures (redeterminations or pseudopolymorphs differing only in the nature of a smaller, co-crystallized molecule). Additional amino acid abbreviations: Abu = aminobutyric acid, Nva = norvaline, nGly = naphtylglycine. With the exception of the last entry, SBLCYS, L-Xaa-L-Xaa amino acids have absolute chirality SS. In the Comment column chirality is indicated if different from this. 
Table S3

Hydrogen bonding patterns in all dipeptide structures

Data set sorted by number of $C(8)$ head-to-tail chains, $N$

\begin{tabular}{|c|c|c|c|c|}
\hline Refcode $^{\mathrm{a}}$ & Seq. ${ }^{b}$ & Stereo $^{c}$ & Solvent/guest/anion & Packing $^{\mathrm{d}}$ \\
\hline \multicolumn{5}{|l|}{$N=0(15)$} \\
\hline BEQJAJ & IW & & dihydrate & $\mathbf{T} 5 * *$ \\
\hline BEQJEN & WV & & dihydrate & $\mathbf{S 5} * *$ \\
\hline BUDXUT & $\mathrm{PE}$ & & dihydrate & $\mathbf{T 5} 5^{* *}$ \\
\hline CAXNUK & $\mathbf{R E}$ & & dihydrate & no layer, $Z^{\prime}=2$ \\
\hline CELTAO10 & YF & & hydrate & $\mathbf{T 5} * *$ \\
\hline CIHNUC & YV & & hydrate & $\mathbf{T 5} * *=$ CELTAO 10 \\
\hline ETONIK & IF & & dihydrate & $\mathbf{T} 5 * *=\mathrm{BEQJAJ}$ \\
\hline GASWEC & $\mathrm{PK}+$ & & acetate & no layer \\
\hline KIXCAW & YW & & hydrate & $\mathbf{T 5} * *=$ CELTAO 10 \\
\hline LAMDEK & HS & & 3.7 hydrate & $\mathbf{T} 4 * *$ \\
\hline MOBYAD & VF & & dihydrate & $\mathbf{T} \mathbf{5}^{* *}=\mathrm{BEQJAJ}$ \\
\hline RAVZEU & $\mathrm{GH}$ & & dihydrate & $\mathbf{T 5} * *$ \\
\hline VEVGOS & $\mathbf{K E}$ & & dihydrate & no layer \\
\hline VIKWUJ & $\mathrm{nVF}$ & & dihydrate & T5 $* *=$ BEQJAJ \\
\hline VUZBAT & RD & & dihydrate & no layer \\
\hline VUZBIB & YL & & hydrate & $\mathbf{T 5}^{* *}=$ CELTAO 10 \\
\hline ZEFZAL10 & YY & & dihydrate & no layer \\
\hline \multicolumn{5}{|l|}{$N=1(27)$} \\
\hline ALGLYL & $\mathrm{AG}$ & & $\mathrm{Li}^{+}, \mathrm{Br}^{-}$,dihydrate & no layer \\
\hline ASPGLY & DG & & hydrate & S4* \\
\hline BEVXEF01 & GD & & dihydrate & no layer \\
\hline CEFGOJ & YE & & hydrate & $\mathbf{T 5}{ }^{*}$ \\
\hline DIYZEQ01 & RD & & hydrate & no layer \\
\hline GLLASP & GN & & & $\left(\mathbf{S 5}^{*}\right)$ \\
\hline GLTLYR10 & GY & & dihydrate & T4** helix \\
\hline GLTRDH01 & GW & & dihydrate & S5 $^{*}$ \\
\hline GLYGCA & GG & & $\mathrm{Ca}^{2+}, 2 \mathrm{Cl}^{-}$ & no layer \\
\hline GLYGLB & GG & & $\mathrm{Li}^{+}, \mathrm{Br}^{-}$ & no layer \\
\hline GLYTRE04 & GT & & dihydrate & anti $=$ GLYGLY04 \\
\hline JADVAL & FP & & hydrate & anti \\
\hline KIXBOJ & SY & & hydrate & S4* \\
\hline KIXBUP & WS & & hydrate & straight chains \\
\hline LACBAS & $\mathrm{GH}+$ & & semisuccinate hydrate & $\mathbf{S} 4 *$ \\
\hline LPROHP20 & $\mathrm{PhP}$ & & hydrate & no layer \\
\hline MAVTAE & nGfG & & $(S)$-methyl lactate clathrate & anti, $Z^{\prime}=2$ \\
\hline MAVTIM & nGfG & & (S)-ethyl pantolactone dimethanol clathr. hydrate & anti $=$ MAVTAE, $Z^{\prime}=2$ \\
\hline MOBYEH & VF & & trihydrate & complex, $Z^{\prime}=4$ \\
\hline POTPET02 & GG & & 1.5 hydrate & S4* \\
\hline QIMBUJ & FA & & dihydrate & screw chains \\
\hline RAVMAC & $\mathrm{HG}+$ & & chloride & straight chains \\
\hline RAVMOQ & LA & & tetrahydrate & $\mathbf{S 5}^{*}$ \\
\hline RAVZIY & $\mathrm{LH}$ & & hydrate & S5* \\
\hline RAVZUK & HE & & & S4* \\
\hline TEJGAQ & $\mathrm{GH}+$ & & chloride dihydrate & S4*, very folded \\
\hline UPUVOR & TfG & & 1-propanol solvate & anti, mimics GLDLPA \\
\hline UPUVUX & TfG & & butan-2-ol solvate & anti, mimics GLDLPA \\
\hline UPUWAE & $\mathrm{TfG}$ & & 2-propanol solvate & anti, mimics GLDLPA \\
\hline UPUWEI & TfG & & cyclohexanol solvate & anti, mimics GLDLPA \\
\hline YAGZOW & II & & dihydrate & $\left(\mathbf{S 5}^{*}\right)$ \\
\hline
\end{tabular}

$N=2(92)$

ALAMET01 AM DL/LD 


\begin{tabular}{|c|c|c|c|c|}
\hline AQAROZ & $\mathrm{AI}$ & & hydrate & VA-class \\
\hline AQARUF & IA & & & VA-class \\
\hline AQASAM & IV & & 0.21 hydrate & VA-class \\
\hline AQASEQ & VI & & 0.22 hydrate & VA-class \\
\hline AQASIU & VV & & hydrate & VA-class \\
\hline BAPBEZ10 & PM & & hydrate & T5 \\
\hline BELCUQ & $\mathrm{EE}$ & & & T5 \\
\hline BIBVOX & PV & & hydrate & T5 \\
\hline BOFZOL & LE & & & S4 \\
\hline BUHGIU & PG & & hydrate & T5 \\
\hline BURLIJ AD & & & & S5 \\
\hline BURLOP & ED & & & S4 \\
\hline CAQTOD & MA & & 2-propanol solvate & $\mathbf{S 4}, Z^{\prime}=2$ \\
\hline CAZGOA01 & VS & & & VA-class, $Z^{\prime}=3$ \\
\hline CIJGUX & VE & & & S5 \\
\hline COCGEG & $\mathrm{AF}$ & & 2 (2-propanol) solvate & S5 \\
\hline COCGIK & VF & & 2-propanol solvate & $\mathbf{S 4}, Z^{\prime}=4$ \\
\hline COCGOQ & $\mathrm{LF}$ & & 0.5 hydrate 2 -propanol solvate & $\mathbf{S 4}, Z^{\prime}=2$ \\
\hline DABQOM & nGfG & & 1,2-dimethoxybenzene clathrate & S4 \\
\hline DEZQOO & $\mathrm{AL}$ & & 0.5 hydrate & T5 \\
\hline DIYZIU & $\mathrm{RE}$ & & hydrate & anti \\
\hline DUHKEW & ED & & hydrate & T4 \\
\hline ETITUW & IL & & 0.91 hydrate & T5-t, $Z^{\prime}=2$ \\
\hline EYIVAJ & SV & & & T5 \\
\hline FABYEM10 & AnV & DL/LD & & S4 \\
\hline FEFYUM & LG & & 0.67 hydrate & unique translation, $Z^{\prime}=3$ \\
\hline FEHPAK & LA & & benzyl methyl sulfoxide clathrate & S4 \\
\hline FEHPEO & LA & & isobutyl methyl sulfoxide clathrate & S4 \\
\hline FOBLUE & VS & & trihydrate & T4 \\
\hline FOBXAW & NV & & 1.33 hydrate & no layer, $Z^{\prime}=3$ \\
\hline FUJZUF & AW & & hydrate & unique $^{f}$ \\
\hline FULGEY02 & WG & & hydrate & T5-t \\
\hline GAGFIG & nVV & & & VA-class \\
\hline GAGFOM & nVL & & & VA-class \\
\hline GAGFUS & nVI & & & VA-class \\
\hline GAGPAI & $\mathrm{BB}$ & & & VA-class \\
\hline GAGPEM & $\mathrm{BnV}$ & & & VA-class \\
\hline GAGPIQ & BV & & & VA-class \\
\hline GAGPOW & $\mathrm{BL}$ & & & VA-class \\
\hline GAGPUC & BI & & & VA-class \\
\hline GAGQAJ & $\mathrm{nVB}$ & & & VA-class \\
\hline GAGQEN & $\mathrm{nVnV}$ & & & VA-class \\
\hline GEHTAP & FW & & 0.75 hydrate & T5-t, $Z^{\prime}=4$ \\
\hline GLUGLY & EG & & & S5 \\
\hline GLYGLY01 & GG & & & T4m ( $\beta$-polymorph) \\
\hline GUFQON06 & GS & & & no layer \\
\hline GUKVUD & SL & & & T5 \\
\hline GUYTAV & nGfG & & 1,2-dimethoxyethane hydrate clathrate & anti \\
\hline HIQWAF & $\mathrm{LL}$ & & 2-methyl-1-propanol solvate & $\mathbf{S 5}, Z^{\prime}=2$ \\
\hline HIZCOJ & LI & & 2.5 hydrate & no layer \\
\hline HUZVON & fGfG & $S R$ & dimethylformamide clathrate & S4 \\
\hline HUZVUT & fGfG & $S R$ & acetamide clathrate & S4 \\
\hline IDUZOW & LL & & 0.87 hydrate & T5-t, $Z^{\prime}=2$ \\
\hline IDUZUC & LF & & 0.86 hydrate & $\mathbf{T 5 - t}, Z^{\prime}=2$ \\
\hline IFABAS & FL & & 1.26 hydrate & T5-t, $Z^{\prime}=2$ \\
\hline IFABEW & $\mathrm{FF}$ & & 2.47 hydrate & T5-t \\
\hline IКОМОМ & LI & & trifluoroethanol & S5 \\
\hline ITOBEA & $\mathrm{F}^{*} \mathrm{~F}^{*}$ & & & $\mathbf{S 5}, Z^{\prime}=3$ \\
\hline ITOBIE & $\mathrm{F}^{*} \mathrm{~F}^{*}$ & & 0.5 trifluoroacetate & anti, $Z$ ' $=2$ \\
\hline ITOBOK & $\mathrm{F}^{*} \mathrm{~F}^{*}$ & & 0.25 trifluoroacetate $0.25 \mathrm{H}_{3} \mathrm{O}^{+} 0.125 \mathrm{H}_{2} \mathrm{O}$ & anti, $Z^{\prime}=2$ \\
\hline
\end{tabular}




\begin{tabular}{|c|c|c|c|c|}
\hline JENTOL01 & fGfG & & $(S)$-isopropylphenylsulfoxide clathrate & S4 \\
\hline JOQLIM & $\mathrm{FF}$ & & 2.0 methanol & S4 \\
\hline JUCSEF01 & LV & & 2-propanol solvate. & S5 \\
\hline JUKMEH & LY & & & S5 \\
\hline JUKMOR & HL & & & T5 \\
\hline JUQQIV & LL & & 1-propanol 2-propanol solvate & anti, $Z^{\prime}=2$ \\
\hline KIXBID & SN & & hydrate & T4 \\
\hline KIYHOP & AS & & & S4m \\
\hline MAVVUA & nGfG & & $-g$ & anti, $Z^{\prime}=2$ \\
\hline MAZXUH & IS & & 0.33 hydrate & T4 \\
\hline MAZYES & MS & & 0.34 hydrate & $\mathbf{T 4}, Z^{\prime}=2$ \\
\hline METMET & MM & & & S4 \\
\hline NAFZID & LV & & 0.75 hydrate & no layer, $Z^{\prime}=4$ \\
\hline NAYJOM & HG & & 0.5 hydrate & no layer, $Z^{\prime}=2$ \\
\hline NAYZET & VA & & 0.33 acetonitrile solvate 0.29 hydrate & VA-class, $Z^{\prime}=3$ \\
\hline NOTSIY & fGfG & & $0.5(R, R)$-bis((o-methylsulfinyl)benzyl)ether clathr. & S4 \\
\hline OKOZAQ & IfG & & methyl phenyl sulfoxide & S4 \\
\hline OKOZEU & LfG & & methyl 3,4-dimethylphenyl sulfoxide & S4 \\
\hline OKOZIY & IfG & & methyl o-tolyl sulfoxide & S5 \\
\hline OLOGEB & MA & & & VA-class, $Z^{\prime}=7$ \\
\hline PAJFIQ & SF & & & T5 \\
\hline PAJPUM & FI & & 0.88 hydrate & $\mathbf{S 5} / \mathbf{T 5}$ hybrid, $Z^{\prime}=2$ \\
\hline PRSARH & PSar & & hydrate & anti \\
\hline RAVZAQ & HM & & & T5 \\
\hline RAVZOE & HD & & trihydrate & T4 \\
\hline RAWBAT & $\mathrm{AH}$ & & ethanol solvate 0.5 hydrate & anti \\
\hline SAMWOT & fGfG & & (4-fluorobenzyl)methylsulfoxide clathrate & S5 \\
\hline SAMWUZ & fGfG & & (4-bromobenzyl)methylsulfoxide clathrate & S5 \\
\hline SBLCYS & $\mathrm{bCbC}$ & & & S5 \\
\hline SEGYOS & $\mathrm{RV}+$ & & acetate & S5 \\
\hline SEYWAU & $\mathrm{KV}+$ & & chloride & S5 \\
\hline SEYWEY & PI & & hydrate & T5 \\
\hline SOJPAI & PY & & hydrate & T5 \\
\hline SUWLIF & LV & & methanol solvate & S5 \\
\hline SUWLOL & LV & & ethanol solvate & $\mathbf{S 5}, Z^{\prime}=4$ \\
\hline TARKUT & $\mathrm{MN}$ & & & S5 \\
\hline TEKNAY & HA & & dihydrate & S5 \\
\hline TELVOV01 & LA & & dimethylsulfoxide clathrate & S4 \\
\hline TIPTOB & VQ & & & S5 \\
\hline TIPTUH & EV & & & T5 \\
\hline VIFFEW & FY & & & S5 \\
\hline WIRYEB & VA & & & VA-class \\
\hline XEGNAY & $\mathrm{FV}$ & & & S5 \\
\hline XEJQOS & fGfG & & rac-benzyl methyl sulfoxide clathrate & $\mathbf{S 4}, Z^{\prime}=2$ \\
\hline XUDVOH & AV & & & VA-class \\
\hline XUDWAU & AV & & 0.25 2-propanol solvate 0.22 hydrate & VA-class, $Z^{\prime}=4$ \\
\hline YAMHUP & $\mathrm{RS}+$ & & acetate hydrate & S4 \\
\hline YICGUM & $\mathrm{KL}+$ & & acetate 0.5 acetic acid solvate 0.5 hydrate & $\mathbf{S 5}, Z^{\prime}=2$ \\
\hline YORPEA & LL & & dimethyl sulfoxide solvate & S5 \\
\hline ZILDON & GQ & & hydrate & S5 \\
\hline This work & VL & & acetonitrile & S5 \\
\hline \multicolumn{5}{|l|}{$N=3(25)^{\mathrm{h}}$} \\
\hline ALAALA & AA & & & T5-t \\
\hline ALAGLY & $\mathrm{AG}$ & & & S5 \\
\hline EMIPAR & $\mathrm{AM}$ & & 0.5 hydrate & S4/T5 hybrid, $Z^{\prime}=2$ \\
\hline ETIWIN & LI & & 0.75 hydrate & no layer, $Z^{\prime}=4$ \\
\hline EWOVAN & AT & & & S5m \\
\hline GLDLPA & GF & $\mathrm{L} / \mathrm{D}$ & & anti \\
\hline GLHPRA & GhP & & & no layer \\
\hline
\end{tabular}




\begin{tabular}{|c|c|c|c|c|}
\hline GLTHRE & GT & $\mathrm{L} / \mathrm{D}$ & hydrate & S5 \\
\hline GLYALB & GA & & & no layer \\
\hline GLYDLA & GA & $\mathrm{L} / \mathrm{D}$ & & no layer \\
\hline GLYGLY04 & GG & & & anti ( $\alpha$-polymorph) \\
\hline GLYLEU10 & GL & & & T5 \\
\hline JAXBUG & LS & & & T5m, tubular \\
\hline JAYFOF & $\mathrm{AB}$ & & & $\mathbf{T 5} / \mathbf{T 5} / \mathbf{T} 4$ hybrid, $Z^{\prime}=3$ \\
\hline LALLSE & AS & & & S4 \\
\hline MAPKOE & TA & & & T4-t \\
\hline MAZYAO & FS & LD & & T5m \\
\hline NIKPOO & $\mathrm{BS}$ & & & T5-t \\
\hline QQQEVJ01 & GF & & & T5 \\
\hline SEHGES & GP & & 0.5 hydrate & unique, $Z^{\prime}=2$ \\
\hline SERGLY & $\mathrm{SG}$ & & & $\mathbf{T} 4 \mathrm{~m}$ \\
\hline WEVWOK & GV & & & $\mathbf{T 5}, Z^{\prime}=7$ \\
\hline WILTIV & fGfG & $S R$ & & T5m \\
\hline XEGHOG & GL & $\mathrm{L} / \mathrm{D}$ & & unique \\
\hline XOSHOC & BA & & & T5-t \\
\hline XOSHUI & $\mathrm{AB}$ & & 0.33 hydrate & $\mathbf{T 5} / \mathbf{T 5} / \mathbf{T 4}$ hybrid, $Z^{\prime}=3$ \\
\hline
\end{tabular}

a Refcode in the CSD (Groom et al., 2016), entries in bold are new since the head-to-tail review (Görbitz, 2010). ${ }^{\mathrm{b}}$ Peptide sequence given with one-letter amino acid abbreviations. Uncommon acids: $\mathrm{nG}=(1$-naphtyl $)$ glycine; $\mathrm{fG}=$ phenylglycine; $\mathrm{nV}=$ norvaline; $\mathrm{B}=2$-amino butyric acid; $\mathrm{hP}=$ hydroxyproline; $\mathrm{bC}=S$-benzylcysteine; Sar $=$ sarcosine $; \mathrm{F}^{*}=$ phenylalanine with $-\mathrm{CH}_{2}-$ replaced by longer chain. An additional ' + ', as in $\mathrm{RS}+$, indicates a peptide net charge of +1 , bold typeface indicates a double zwitterion.

c Stereochemistry is given when deviating from LL, DD, L or D. L/D or DL/LD indicate racemates. An L-amino acid usually has absolute chirality $S$.

d The basic pattern is given when present. A succeeding ' $\mathrm{m}$ ', as in $\mathbf{T 5}$ m, indicates a modified pattern. One or two succeeding stars indicates one or two missing $C(8)$ chains, parentheses surround tentative classifications.

${ }^{\mathrm{f}}$ unique $=$ parallel $\mathrm{H}$ bond pattern occurring in this structure only.

g $0.5(S)$-methyl 2-hydroxy-3,3-dimethylbutyrate methanol clathrate 0.5 hydrate.

${ }^{\mathrm{h}}$ When $Z$ ' $>1$, the high value for $N$ may not apply to all molecules. 


\section{Table S4}

Torsion angles $\left({ }^{\circ}\right)$ in dipeptide structures for the main structural classes

$\begin{array}{ll} & \text { CSD refcode and torsion angle as defined in Scheme 1 } \\ \text { Inverted from D-Xaa-D-Xaa structure } \\ \text { Extreme value within group } \\ \text { Average value and sample standard deviation } \\ \text { ABCDEF } & \text { Representative molecule in group (Fig. 7) }\end{array}$

S4

\begin{tabular}{|c|c|c|c|c|}
\hline Refcode & $\psi_{1}$ & $\omega_{1}$ & $\varphi_{2}$ & $\psi \mathrm{T}$ \\
\hline COCGIK & 132,4 & 173,0 & $-150,9$ & 20,0 \\
\hline COCGIK & 121,9 & 172,5 & $-160,0$ & $-11,6$ \\
\hline COCGIK & 130,9 & 173,8 & $-149,4$ & 19,5 \\
\hline COCGIK & 122,3 & 172,9 & $-159,3$ & $-8,9$ \\
\hline FEHPEO & 136,2 & 178,1 & $-159,0$ & $-14,1$ \\
\hline FEHPAK & 129,3 & 177,9 & $-162,6$ & $-17,4$ \\
\hline COCGOQ & 127,9 & 175,3 & $-152,6$ & 17,5 \\
\hline COCGOQ & 124,9 & 176,0 & $-159,0$ & $-5,9$ \\
\hline JOQLIM & 162,4 & 170,3 & $-153,4$ & 14,5 \\
\hline CAQTOD & 131,8 & 174,5 & $-158,5$ & $-13,2$ \\
\hline CAQTOD & 141,3 & 171,5 & $-140,1$ & 11,6 \\
\hline METMET & 133,1 & 179,9 & $-149,0$ & $-10,3$ \\
\hline OKOZAQ & 153,8 & 178,8 & $-166,1$ & $-15,8$ \\
\hline OKOZEU & 144,9 & 184,8 & $-159,6$ & $-24,8$ \\
\hline DABQOM & 131,5 & 175,1 & $-146,2$ & $-32,5$ \\
\hline JENTOLO1 & 152,4 & 169,9 & $-145,5$ & $-19,9$ \\
\hline NOTSEU & 148,1 & 168,0 & $-140,2$ & $-21,7$ \\
\hline NOTSIY & 145,1 & 184,9 & $-159,8$ & $-18,6$ \\
\hline LALLSE & 124,8 & 182,0 & $-156,9$ & $-1,6$ \\
\hline BOFZOL & 135,5 & 172,3 & $-160,3$ & $-18,1$ \\
\hline BURLOP & 134,3 & 174,3 & $-137,7$ & $-32,2$ \\
\hline TELVOV01 & 137,0 & 175,1 & $-152,5$ & $-12,6$ \\
\hline RUGHIK & 165,1 & 176,7 & $-168,1$ & $-23,6$ \\
\hline XEJQOS & 141,3 & 183,4 & $-155,0$ & $-29,1$ \\
\hline XEJQOS & 140,0 & 178,3 & $-144,6$ & $-34,1$ \\
\hline XEJQEI & 143,8 & 172,8 & $-146,1$ & $-20,2$ \\
\hline XEJQIM & 139,1 & 185,1 & $-158,6$ & $-18,3$ \\
\hline Average & 138,2 & 176,2 & $-153,7$ & $-11,9$ \\
\hline St.dev. & 11,2 & 4,7 & 8,0 & 15,9 \\
\hline
\end{tabular}

T4

\begin{tabular}{lrrrr} 
Refcode & $\boldsymbol{\psi}_{1}$ & $\omega_{1}$ & $\boldsymbol{\varphi}_{2}$ & $\boldsymbol{\psi}_{\mathrm{T}}$ \\
\hline DUHKEW & 148,0 & 161,3 & $-118,2$ & $-31,9$ \\
\hline FOBLUE & 134,2 & 174,7 & $-160,2$ & 0,4 \\
\hline KIXBID & 124,4 & 187,7 & $-153,0$ & $-7,4$
\end{tabular}




\begin{tabular}{lrrrr} 
MAZXUH & 136,4 & 175,3 & $-162,8$ & $-17,1$ \\
MAZYES & 145,5 & 168,1 & $-164,8$ & $-8,0$ \\
MAZYES & 132,0 & 178,3 & $-162,0$ & $-15,4$ \\
RAVZOE & 115,3 & 172,7 & $-143,4$ & 0,0 \\
\hline Average & 133,7 & 174,0 & $-152,1$ & $-11,3$ \\
\hline St. dev. & 11,4 & 8,2 & 16,7 & 11,3 \\
\hline
\end{tabular}

S5

\begin{tabular}{lrrrr} 
Refcode & $\boldsymbol{\psi} \mathbf{1}$ & $\boldsymbol{\omega} \mathbf{1}$ & $\boldsymbol{\varphi} \mathbf{2}$ & $\boldsymbol{\psi} \mathbf{T}$ \\
\hline ITOBEA & 168,6 & 172,7 & $-72,9$ & $-33,7$ \\
ITOBEA & 168,7 & 166,7 & $-70,0$ & $-22,7$ \\
ITOBEA & 168,1 & 163,6 & $-71,5$ & $-31,8$ \\
BURLIJ & 175,8 & 175,9 & $-113,6$ & 71,3 \\
CIJGUX & 124,5 & 175,2 & $-81,7$ & $-30,7$ \\
COCGEG & 159,9 & 171,4 & $-77,6$ & $-19,5$ \\
GLUGLY & 166,1 & 175,6 & $-74,2$ & $-27,1$ \\
HIQWAF & 168,5 & 174,9 & $-127,0$ & $-45,5$ \\
HIQWAF & 128,8 & 168,4 & $-86,1$ & $-29,7$ \\
IKOMOM & 117,8 & 173,2 & $-65,5$ & $-41,1$ \\
IKOMOM & 162,6 & 168,6 & $-99,5$ & $-52,9$ \\
JUCSEF01 & 156,5 & 179,4 & $-126,7$ & $-71,6$ \\
JUKMEH & 163,8 & 158,8 & $-110,9$ & $-41,5$ \\
OKOZIY & 132,4 & 171,9 & $-93,0$ & $-22,0$ \\
\hline SAMWOT & 147,0 & 170,2 & $-101,6$ & $-55,4$ \\
SAMWUZ & 154,8 & 167,1 & $-106,1$ & $-43,8$ \\
\hline SBLCYS & 148,0 & 174,4 & $-114,0$ & 87,7 \\
\hline SEGYOS & 163,1 & 172,4 & $-91,4$ & $-28,9$ \\
SEYWAU & 129,3 & 176,5 & $-90,1$ & $-27,7$ \\
SUWLIF & 153,0 & 169,9 & $-116,5$ & $-64,8$ \\
SUWLOL & 151,0 & 162,9 & $-87,6$ & $-54,8$ \\
SUWLOL & 151,1 & 168,1 & $-85,2$ & $-56,1$ \\
SUWLOL & 151,5 & 162,3 & $-88,4$ & $-54,3$ \\
SUWLOL & 150,2 & 168,5 & $-84,8$ & $-55,2$ \\
TARKUT & 155,5 & 172,0 & $-95,7$ & $-29,4$ \\
TEKNAY & 156,8 & 164,9 & $-109,6$ & 74,2 \\
TIPTOB & 141,6 & 169,2 & $-101,2$ & 74,9 \\
VIFFEW & 161,1 & 158,6 & $-106,1$ & $-39,1$ \\
\hline XEGNAY & 128,9 & 178,8 & $-109,6$ & $-80,9$ \\
\hline YICGUM & 150,4 & 165,9 & $-74,5$ & $-27,4$ \\
YICGUM & 152,2 & 167,4 & $-78,8$ & $-26,3$ \\
YORPEA & 139,1 & 166,1 & $-149,5$ & $-14,5$ \\
ZILDON & 165,2 & 180,4 & $-115,3$ & $-41,1$ \\
\hline This WOrk & 140,9 & 170,6 & $-95,9$ & $-11,7$ \\
\hline Average & 151,6 & 170,1 & $-96,2$ & $-25,7$ \\
\hline St. deV. & 14,5 & 5,5 & 19,2 & 41,3 \\
\hline & & & &
\end{tabular}


T5

\begin{tabular}{lrrrrr} 
Refcode & $\boldsymbol{\psi}_{\mathbf{1}}$ & $\boldsymbol{\omega}_{\mathbf{1}}$ & $\boldsymbol{\varphi}_{\mathbf{2}}$ & $\boldsymbol{\psi} \boldsymbol{\Psi}_{\mathbf{T}}$ \\
\hline BAPBEZ10 & 167,5 & 168,8 & $-68,0$ & $-22,2$ \\
BELCUQ & 155,6 & 167,6 & $-94,8$ & $-34,4$ \\
BIBVOX & 167,3 & 168,5 & $-73,9$ & $-24,4$ \\
BUHGIU & 168,2 & 169,6 & $-74,6$ & $-18,9$ \\
DEZQOO & 163,3 & 168,3 & $-64,9$ & $-23,7$ \\
EYIVAJ & 152,6 & 157,4 & $-63,7$ & $-24,7$ \\
GUKVUD & 155,0 & 158,0 & $-68,2$ & $-19,1$ \\
JUKMOR & 164,5 & 174,8 & $-78,2$ & $-18,7$ \\
PAJFIQ & 154,2 & 157,5 & $-66,2$ & $-21,2$ \\
GLYLEU10 & 171,6 & 168,7 & $-64,9$ & $-30,2$ \\
RAVZAQ & 160,6 & 175,0 & $-77,6$ & $-17,5$ \\
SEYWEY & 166,3 & 170,6 & $-75,8$ & $-22,4$ \\
\hline SOJPAI & 162,2 & 174,4 & $-67,9$ & $-34,9$ \\
TIPTUH & 156,4 & 173,7 & $-75,1$ & $-14,6$ \\
\hline QQQEVJ01 & 166,1 & 169,2 & $-66,3$ & $-28,8$ \\
\hline Average & 162,1 & 168,1 & $-72,0$ & $-23,7$ \\
\hline St. dev. & 6,0 & 6,0 & 8,0 & 6,0 \\
\hline N-term Pro & & & &
\end{tabular}

T5-t

\begin{tabular}{lrrrrr} 
Refcode & $\boldsymbol{\psi}_{\mathbf{1}}$ & $\boldsymbol{\omega}_{\mathbf{1}}$ & $\boldsymbol{\varphi}_{\mathbf{2}}$ & $\boldsymbol{\psi}_{\boldsymbol{T}}$ \\
\hline ETITUW & 136,8 & 174,6 & 49,9 & 47,9 \\
\hline ETITUW & 130,9 & 185,5 & 50,4 & 49,3 \\
FULGEY02 & 156,5 & 177,0 & 60,2 & 31,8 \\
\hline GEHTAP & 144,6 & 181,0 & 56,0 & 29,5 \\
GEHTAP & 117,9 & 178,0 & 51,2 & 41,8 \\
GEHTAP & 106,6 & 185,8 & 55,1 & 33,3 \\
GEHTAP & 108,4 & 188,7 & 51,2 & 39,1 \\
IDUZOW & 129,6 & 174,9 & 47,9 & 50,6 \\
IDUZOW & 127,8 & 177,9 & 51,0 & 51,7 \\
IDUZUC & 125,0 & 179,9 & 47,6 & 52,6 \\
\hline IDUZUC & 124,4 & 185,7 & 49,2 & 54,1 \\
\hline IFABAS & 152,6 & 183,1 & 52,5 & 39,4 \\
IFABAS & 155,3 & 183,0 & 51,9 & 44,9 \\
IFABEW & 157,8 & 180,9 & 55,4 & 43,7 \\
\hline Average & 133,9 & 181,1 & 52,1 & 43,6 \\
\hline St. dev. & 17,3 & 4,4 & 3,5 & 8,1 \\
\hline
\end{tabular}

T5**

\begin{tabular}{|l|r|rrr|}
\hline Refcode & $\boldsymbol{\psi}_{1}$ & $\omega_{1}$ & $\boldsymbol{\varphi}_{\mathbf{2}}$ & $\boldsymbol{\psi}_{\mathbf{T}}$ \\
\hline BUDXUT & 160,3 & 173,8 & $-95,3$ & $-34,9$ \\
\hline CELTAO10 & 164,7 & 177,4 & $-70,6$ & $-34,9$ \\
\hline CIHNUC & 164,6 & 176,7 & $-79,3$ & $-45,5$ \\
\hline KIXCAW & 161,6 & 177,3 & $-67,6$ & $-35,2$
\end{tabular}




\begin{tabular}{lrrrr} 
RAVZEU & 164,1 & 182,2 & $-103,3$ & $-2,2$ \\
VUZBIB & 161,8 & 179,0 & $-83,5$ & $-35,3$ \\
\hline Average & 162,8 & 177,7 & $-83,3$ & $-31,3$ \\
\hline St. dev. & 1,8 & 2,8 & 13,9 & 14,9 \\
\hline
\end{tabular}

N-term Tyr

\begin{tabular}{|c|c|c|c|c|}
\hline \multicolumn{5}{|l|}{$\mathrm{T} 5 * *(t)$} \\
\hline Refcode & $\psi_{1}$ & $\omega_{1}$ & $\varphi_{2}$ & $\psi_{\top}$ \\
\hline BEQJAJ & 147,5 & 166,0 & 51,9 & 49,7 \\
\hline ETONIK & 149,9 & 170,7 & 49,5 & 48,3 \\
\hline MOBYAD & 151,3 & 172,3 & 48,5 & 48,4 \\
\hline VIKWUJ & 166,5 & 171,5 & 55,0 & 37,2 \\
\hline Average & 153,8 & 170,1 & 51,2 & 45,9 \\
\hline St. dev. & 8,6 & 2,8 & 2,9 & 5,9 \\
\hline
\end{tabular}

S4*

$\begin{array}{lrrrr}\text { Refcode } & \boldsymbol{\psi}_{1} & \omega_{1} & \boldsymbol{\varphi}_{2} & \psi_{\boldsymbol{\tau}} \\ \text { ASPGLY } & 147,101 & 171,643 & 152,782 & -5,098 \\ \text { KIXBOJ } & 171,43 & 169,175 & -148,633 & 6,137 \\ \text { LACBAS } & -164,92 & 175,075 & -116,875 & -17,001 \\ \text { POTPET02 } & -174,711 & 172,753 & 160,556 & -20,944 \\ \text { RAVZUK } & 159,64 & 160,749 & -71,066 & -11,42\end{array}$

\section{References}

Akazome, M., Senda, K. \& Ogura, K. (2002). J. Org. Chem. 67, 8885-8889.

Akazome, M., Ueno, Y., Ooiso, H. \& Ogura, K. (2000). J. Org. Chem. 65, 68-76.

Biswas, A. B., Hughes, E. W., Sharma, B. D. \& Wilson, J. N. (1968). Acta Cryst. B24, 40-50.

Groom, C. R., Bruno, I. J., Lightfoot, M. P. \& Ward, S. C. (2016). Acta Cryst. B72, 171-179.

Görbitz, C. H. (2001). Acta Cryst. C57, 575-576.

Görbitz, C. H. (2004a). Acta Cryst. B60, 569-577.

Görbitz, C. H. (2004b). Acta Cryst. C60, o810-0812.

Görbitz, C. H. (2010). Acta Cryst. B66, 84-93.

Kvick, A., Al-Karaghouli, A. R. \& Koetzle, T. F. (1977). Acta Cryst. B33, 3796-3801.

Mason, T. O., Chirgadze, D. Y., Levin, A., Adler-Abramovich, L., Gazit, E., Knowles, T. P. J. \& Buell, A. K. (2014). ACS Nano 8, 1243-1253.

Murali, R., Lalitha, V., Subramanian, E. \& Parthasarathy, R. (1986). Int. J. Pept. Protein Res. 27, 160-164. 